\title{
Symptom Proximity in Diagnostic Problem
}

\author{
Otakar Kříž
}

Prague, Czech Republic

Copyright (c) 2017 by authors, all rights reserved. Authors agree that this article remains permanently open access under the terms of the Creative Commons Attribution License 4.0 International License

\begin{abstract}
An algorithm SP (= Symptom Proximity) is suggested for solving discrete diagnostic problem. It is based on probabilistic approach to decision-making under uncertainty, however, it does not use knowledge integration from marginal distributions.
\end{abstract}

Keywords Probabilistic Decision Making, Diagnostic Problem

\section{Informal analysis}

The ability of decision-making belongs to the most important mechanismus build in complex organisms. Optimal strategies for decision-making determine the success or even survival of different animal species (Darwin theory), trading companies or individual patients.

As mentioned e.g. in [5], decision-making can take place on a purely intuitive basis (i.e. an individual decision is provided by a genetically inborn mechanism or it can be given by experience acquired during the life span of a decision-maker and stored in neural structures of his brain ). Or, decisionmaking can take place on the basis of a more considered strategy and in the framework of a formalized theory.

When collecting a large number of facts relating to a situation, there appears a point, in certain phase, where facts are "abstracted" to a piece of knowledge. (The process is refered to as hegelian dialectic concept of "quantitative change leads to qualitative change".) Knowledge is usually expressed in form of sets of implications. Then, in the context of decisionmaking, observed facts (called evidences ) on an individual object are used as antecedents and the required decision is, hopefully, provided via laws of logics from the succedents (of the implications). However, even this approach involves implicitely certain degree of uncertainty (e.g. when to switch from facts to a law/knowledge).

The tolerable precision is domain dependent. In physics, a theory must explain data with precision to six decimal positions. In soft sciences, models are less demanding.

Therefore, one can generalize that decision-making cannot be separated from uncertainty. Partially, it is due to the men- tioned "ever present lack of data" and partially, there exists an "uncertainty" which of formal theories of uncertainty to use as a model. Even in probability, taken usually as a normative theory (for uncertainty), there exist alternatives (see e.g.[4]).

One of the most fruitful lines in probabilistic decisionmaking (see subsection 2.1) can be divided into four phases:

First, select less-dimensional distributions, considered as marginals of a theoretical joint distribution, as input knowledge.

Second, one can construct (integrate) an explicit formula for the joint distribution. It can be done via strong assumptions like conditional independence between marginals expressed as graph models .

Third, given an evidence about an object (i.e. measured on the object), the formula is reduced (marginalized) to a simpler form where the evidence can be directly applied.

Fourth, the "best" decision is the one that yields largest conditional probability on diagnosis variable (i.e. containing decisions) given the observed evidence.

The topic of this paper is a method/algorithm SP that does not use the marginals (and assumptions about their conditional independence) and finds an approximation of the largest conditional probability directly from the data (called statistical file in the sequel and defined in subsection 2.3).

Let us suppose that both models (i.e. "marginal "one and SP) are finite and discrete.

( Explicitly, we do not consider e.g. family of log-linear distributions and no estimation of their parameters from the statistical file takes place as is usual in standard statistics).

There is a discrete number of symptom variables, discrete number of symptoms in range of each symptom variable, discrete number of diagnoses/decisions and finite number of objects in the statistical file (representing knowledge).

It should be stressed that if one uses the term "measured" it should be interpreted as "measured and discretized". E.g. biochemical values, though continuous, are reduced to dichotomies (greater or less than a threshold) or trichotomies ("standard values", "too low","too high").

This finiteness and discretization need not be a limiting factor, as in many decision-making situations, there is small number of alternatives, as well. In most frequent situations, the decision-making takes place on the basis of not too many 
symptom variables.

In "marginal approach", the joint probability formula is integrated once forever from marginals (elicited, in their turn, from a "learning" statistical file ).

Then, this unique formula is used for each evidence available for the object/patient/case.

Whereas, "marginal models" construct an unique approximative distribution of an unique theoretical joint distribution, the SP-model uses for decision-making an adhoc approximation of conditional probability ( derived from the unique theoretical joint distribution). (This link is established by the supposition that the statistical file was generated (by nature?) according to a theoretical joint distribution.) This adhoc approximation of conditional probability is generated freshly from scratch for each set of symptom variables (carrying evidence measured on the object/patient). (In other words, the term adhoc relates to a disclosed set of symptom variables.)

The aim of SP is not to "predict" the shape of aposteriori probabilities of all considered diagnoses, but only to find the most probable diagnosis/decision and even the numerical value of its aposteriori probability is not important. In other words, what matters is just the first place in this ranking.

What is new?

In eighties, it was believed that whereas the data (i.e. statistical file) is not sufficient for estimating the joint distribution, it should do for less-dimensional tables (i.e. supposed marginals). Moreover, these marginals were considered as given externally and reliable as "incorporated truth". Beside being given from outside, the marginals were just few. One might use a marginal for integration or leave it out. Not ask for new ones! The argument that marginals cannot be obtained otherwise than from the data was not paid sufficient attention to. If it would have been taken into consideration, one could ask for more marginals, but, at the same time, it would raise questions like "How many marginals should we ask for?" and "What would be the best composition of those marginals?".

The central notion was the approximation of the joint distribution whereas it is the approximation of conditional probability that SP takes as its "flag ship". At the same time, the role of disclosed symptom variables carrying evidence is stressed for SP, as well. Then, the detour "integration to joint distribution" and subsequent marginalization is not needed any more.

A specific methodology based on proximity (of vector of evidences and respective vectors constructed from cases in the statistical file) in space of symptom variables is used in SP algorithm.

To explain the theory of the SP algorithm, simple metrics is introduced in section 3 .

As details matter and redundancy is preferable to ambiguity, the essence of the algorithm SP is described twice in section 4.2. Namely, via a flowchart (see Figure 1.) and via a code written in a symbolic language. The latter description makes possible to derive computational complexity for SP in section 5 .

However, the real strength of SP lies in the fact that this approach is fast enough to operate on large statistical files with large number of symptom variables.

As far as decision quality is concerned, the situation is not so transparent. On one hand side, it is possible to construct examples where SP dominates the "marginal approach". It may happen when input marginals are "at wrong position" to the disclosed symptom variables carrying evidences. However, in general, the dominance of SP cannot be directly proved and one can only compare SP and one of many possible "marginal" algorithms, with different sets of marginals and for different evidence-carrying symptom variables. And it has to be done in many combinations. This "branching expansion" is multiplied by two when we consider two testing techniques. First, "testing" and "learning" statistical files are identical. Second, the "leave one out" technique, described in section 6 , is used.

The final impression (from this multicriterial decisionmaking) is that SP does very well. It is based on experience from many comparative runs. If it is not dominant, SP belongs to Pareto optimum, at least.

It should be noted that SP does not use the empirical distribution derivable from the statistical file. (The "mass" of the empirical distribution is concentrated only in the atoms of the defining set algebra that appears in cases from the statistical file and it is zero anywhere else).

One may ask why this line of research (i.e. "without marginals") was not followed as a main stream before?

In my opinion, during the last decades, external material conditions (computerization and existence of large databases) changed a lot. In the meantime, the original paradigm was refined and improved by many researchers. The model became so rich, successful and invested in that the inertia prevented an investigation if basic suppositions cannot be weakened.

\section{Specific statement of the problem}

\subsection{Historical background}

Firat attempts for machine-assisted decision-making under uncertainty are marked by rule-based expert systems Mycin and Prospector in early eighties. Weights in rules were interpreted as conditional probabilities. But the way the rules were combined was not a probabilistic one. The same held for systems with fuzzy number approach. At that time, Albert Perez (in [11]) raised the requirement that partial knowledge should be "integrated" intensionally i.e. using the concept of theoretical joint distribution $P$. Knowledge was understood as probability or conditional probability elicited either from experts or observed from experiments. The best way to keep it, at least partially, complete and homogenous was to assume that it comes in form of less-dimensional distributions that were supposed to be marginals of the theoretical joint $P$. Thanks to smaller sizes, marginals could be estimated from available data. The main effort in the subsequent research was concentrated on the way how to assemble effective approximations of the joint $P$. The formulation of the task was known as marginal problem already in [6] and its 
specific solution was suggested even before in [2]. Different models, connected with names like Lauritzen, Spiegelhalter, Dempster, Shafer, Pearl, Dawid, were studied with assumptions about conditional independence of variables appearing in $P$ that helped to integrate the marginals. At present, there exist professional software packages (e.g. Hugin) supporting the decision-making on commercial basis. As, beside different algorithms, even the selection of proper marginals may be a problem of its own (see e.g. [9] and [10]), this paper tries to study an alternative to the marginal approach.

\subsection{The layout of the paper}

1. Informal (i.e. without notation) analysis of the diagnostic problem in probabilistic setting is given in section 1 .

2. There are historical reminiscences explaining the position of the suggested method in a broader context in subsection 2.1 .

3. Basic notions are defined including the formulation of the diagnostic problem and describing the role of the statistical file $\mathcal{F}$ in subsection 2.3 .

4. The essential features of the algorithm $S P$ are laid down in section 3 .

5. Realisation of $S P$ is described in section 4 via a flowchart (in subsection 4.1) and via symbolic programming language (in subsection 4.2).

6. On the basis of the latter description, computational complexity $C_{S P}$ of $S P$ in terms of "length" $l=|\mathcal{F}|$ of the file $\mathcal{F}$ and of its "width" $n=\left|\left\{\xi_{1}, \xi_{2}, \cdots \xi_{n}\right\}\right|$ is estimated and verified experimentally for different values of $l$ and $w$ in section 5 .

7. "Discernment power" of $S P$ (i.e. absolute values or percentage of wrong classifications ) is tested for different "apertures" ( sets of symptom variables whose values are disclosed to $S P$ as evidence). Testing is performed both via method "leave one out" as well as on all data in simulation runs. The results are compared with a simple marginal-based algorithm under the same testing conditions in section 6 .

8. In section 7, there are features of $S P$ sorted as "pros" and "cons". Then, there are two open questions suggested for further investigation and finally, there is a summarizing conclusion.

\subsection{Basic notions and notations}

Let $(\Omega, \mathcal{X}, P)$ be a probabilistic space, $\boldsymbol{\eta}=\boldsymbol{\xi}_{\mathbf{0}}, \boldsymbol{\xi}_{\mathbf{1}}, \boldsymbol{\xi}_{\mathbf{2}}, \ldots \boldsymbol{\xi}_{\boldsymbol{n}}$ be finite sets and $\xi_{r}:(\Omega, \mathcal{X}, P) \longrightarrow\left(\boldsymbol{\xi}_{r}, 2^{\boldsymbol{\xi}_{r}}\right)$ for $r=$ $0,1,2, \cdots n$ be measurable functions.

Though the topic is defined in a formal way, the names of objects in the universe of discussion (e.g. diagnosis, symptoms etc.) are taken from the field of medicine to give them a semantical interpretation and ease up understanding of basic notions and character of their interaction.

The mutual behaviour of all random variables $\eta, \xi_{1}, \xi_{2} \cdots \xi_{n}$ is described by a theoretical joint probability distribution $P_{\eta \xi_{1} \xi_{2} \cdots \xi_{n}}$.

Decision making under uncertainty with probabilistic background can be interpreted as the diagnostic problem with the following formulation:

Diagnostic problem: Find the diagnosis $d\left(s_{1}, s_{2} \cdots s_{n}\right) \in \boldsymbol{\eta}$ that is the most probable (according to the $P_{\eta \xi_{1}, \xi_{2} \cdots \xi_{n}}$ ) on the set $\left\{\omega \in \Omega \mid \xi_{1}(\omega)=s_{1} \& \xi_{2}(\omega)=s_{2} \& \cdots \xi_{n}(\omega)=s_{n}\right\}$ for a given (i.e. observed) arbitrary combination $\left(s_{1}, s_{2} \cdots s_{n}\right)$ of values of symptom variables from the cartesian product $\boldsymbol{\Xi}=\boldsymbol{\xi}_{\mathbf{1}} \times \boldsymbol{\xi}_{\mathbf{2}} \times \ldots \boldsymbol{\xi}_{\boldsymbol{n}}$. If we wish to predict the values of diagnostic variable $\eta$, the conditional probability $P_{\eta \mid \xi_{1} \xi_{2} \ldots \xi_{n}}$ (derivable from $P_{\eta \xi_{1} \xi_{2} \ldots \xi_{n}}$ ) should be used instead of $P_{\eta} \xi_{1} \xi_{2} \ldots \xi_{n}$.

Optimal decision: The value of diagnosis $d$ from $\boldsymbol{\eta}$ that should be selected if the values of symptom variables are $\left(s_{1}, s_{2} \cdots s_{n}\right)$ to keep the wrong classifications of $d$ as low as possible), called Bayes solution, is for each $\left(s_{1}, s_{2} \cdots s_{n}\right) \in \boldsymbol{\xi}_{\mathbf{1}} \times \boldsymbol{\xi}_{\mathbf{2}} \times \ldots \boldsymbol{\xi}_{\boldsymbol{n}}$

given by the formula

$$
d_{o p t}\left(s_{1}, s_{2} \cdots s_{n}\right)=\underset{d \in \boldsymbol{\eta}}{\operatorname{argmax}} P_{\eta \mid \xi_{1} \xi_{2} \ldots \xi_{n}}\left(d \mid s_{1}, s_{2} \cdots s_{n}\right)
$$

So far the theory. Unfortunately, in the "real world", we are never given the theoretical distribution $P_{\eta \xi_{1} \xi_{2} \ldots \xi_{n}}$ in full and directly. To compensate for this, we expect to have some indirect information about $P_{\eta \xi_{1} \xi_{2} \cdots \xi_{n}}$ that will be called knowledge base and denoted by $\mathcal{K}$. It is done by postulating a set of conditions that we believe the theoretical $P_{\eta \xi_{1} \xi_{2} \ldots \xi_{n}}$ fulfills.

Marginal problem: Using the concept of marginal problem, see [6], knowledge base $\mathcal{K}$ is given as a set of "lowdimensional" distributions (i.e. number of variables in the distribution does not exceed e.g. 10. ), postulated as theoretical marginal distributions of the $P_{\eta \xi_{1}, \xi_{2} \ldots \xi_{n}}$. Beside the marginals, there are usually made assumptions about conditional independence holding between groups of random variables. It is interesting that the topic was so attractive that it was addressed in several waves, usually after 20 years. Original and interesting ideas were not just the product of the last two decades but go back much deeper. See e.g. [2], [6],[3], [1]. Instead of the unknown $P_{\eta \xi_{1} \xi_{2} \cdots \xi_{n}}$, we try to construct (from the marginals) its suitable approximation $\hat{P}_{\eta \xi_{1} \xi_{2} \cdots \xi_{n}}$ that could play its role in the formula (1).

If existence of marginals is postulated, it is natural to ask where do they come from. Therefore, another notion should be specified.

Statistical file F: Let $\left(\omega_{1}, \omega_{2}, \cdots \omega_{s}\right)$ be a sequence, where individual $\omega_{i} \in \Omega$ denote realizations of a random selection 
from $\Omega$,

then the sequence $\left(\eta\left(\omega_{l}\right), \xi_{1}\left(\omega_{l}\right), \xi_{2}\left(\omega_{l}\right) \cdots \xi_{n}\left(\omega_{l}\right)\right)_{l=1}^{s}$ of points in cartesian product $\boldsymbol{\eta} \times \boldsymbol{\xi}_{\mathbf{1}} \times \boldsymbol{\xi}_{\mathbf{2}} \times \ldots \boldsymbol{\xi}_{\boldsymbol{n}}$ is a statistical file $\mathrm{F}$ of size $s$ (i.e. $s=|\mathcal{F}|$ ) and $(\mathcal{F})_{r}$ is the $r$-th member of the sequence $\mathcal{F}$.

There exists a taciturn assumption that decision making about a concrete case (patient) should be very fast (about $1 \mathrm{sec} /$ pers.). On the other hand, longer time (e.g. hours of CPU time) devoted to selecting and populating the marginals (in the learning phase) is tolerable. This may be one of the reasons why the "marginal approach" is the standard way.

However, using marginals for "integrating" $\hat{P}_{\eta \xi_{1} \xi_{2} \cdots \xi_{n}}$ and its subsequent conditioning need not be mandatory for solving the diagnostic problem.

\section{Basic idea of SP algorithm}

An algorithm, called SP ( = Symptom Proximity), tries to construct necessary conditional probabilities directly from available statistical data file $\mathcal{F}$. Basic idea of SP can be explained by the assumption "Patients with similar symptoms should have a similar diagnosis". Hence, the name of the algorithms SP interpretes the similarity as a proximity in the sense of a very natural metrics.

\section{Proximity metrics $\rho$ :}

$$
\rho: \Xi \times \Xi \longrightarrow \boldsymbol{R} \quad(\mathbf{u}, \mathbf{v}) \longmapsto n-\sum_{i=1}^{n} \delta\left((\mathbf{u})_{i},(\mathbf{v})_{i}\right)
$$

where $\delta(\cdot, \cdot)$ is the Kronecker function and $(\mathbf{u})_{i}$ is the $\mathrm{i}$-th component of the sequence $\mathbf{u}$ The mapping $\rho$ is a metrics (i.e. reflexivity, symmetry, triangular inequality) on $\boldsymbol{\Xi}$ that can be used for defining equivalence classes on $\boldsymbol{\Xi}$. For each fixed $\mathbf{v} \in \boldsymbol{\Xi}$, there exist $n+1$ sets $C_{0}(\mathbf{v}), C_{1}(\mathbf{v}), \cdots C_{n}(\mathbf{v})$, where $C_{k}(\mathbf{v})=\{\mathbf{u} \in \boldsymbol{\Xi} \mid \rho(\mathbf{u}, \mathbf{v})=k\}$.

The next step is to estimate $P\left(C_{k}(\mathbf{v})\right)$. It can be done, in a natural way, using available data (i.e. the statistical file $\mathcal{F}$ ).

$$
P\left(C_{k}(\mathbf{v})\right)=\sum_{j=1}^{|\mathcal{F}|} \delta\left(\rho\left(\left((\mathcal{F})_{j}\right) \mathbf{\Xi}, \mathbf{v}\right), k\right) /|\mathcal{F}|
$$

where $(\mathcal{F})_{j}$ is the j-th vector from file $\mathcal{F}$ i.e. $(\mathcal{F})_{j} \in \boldsymbol{\eta} \times \boldsymbol{\Xi}$ Similarly, $\left((\mathcal{F})_{j}\right)_{\boldsymbol{\Xi}}$ is that part of the $\mathrm{j}$-th vector $(\mathcal{F})_{j}$ that corresponds to symptom variables i.e. $\left((\mathcal{F})_{j}\right)_{\Xi} \in \boldsymbol{\Xi}$. We are interested in the set $C_{k}(\mathbf{v})$ with smallest $k$ but at the same time such that $P\left(C_{k}(\mathbf{v})\right)>0$. Let us denote this optimal $k$ as $k_{0}$

Finally, the conditional probability $P \eta \mid C_{k_{0}}(\mathbf{v})(d \mid \mathbf{v})$ of $\eta$ on $C_{k_{0}}(\mathbf{v})$ can be defined.

To shorten the shape of the final expression for the $P \eta \mid C_{k_{0}}(\mathbf{v})(d \mid \mathbf{v})$, an auxillary variable $D\left(\mathcal{F}, \mathbf{v}, k_{0}, d\right)$ will be introduced. It denotes the number of cases (patients) from the statistical file $\mathcal{F}$ that "belong" to the equivalence class $C_{k_{0}}(\mathbf{v})$ and at the same time they have the diagnosis $d$

$$
D\left(\mathcal{F}, \mathbf{v}, k_{0}, d\right)=\sum_{j=1}^{|\mathcal{F}|} \delta\left(\rho\left(\left((\mathcal{F})_{j}\right) \mathbf{\Xi}, \mathbf{v}\right), k_{0}\right) \delta\left(\left((\mathcal{F})_{j}\right)_{\eta}, d\right)
$$

(Similarly according to the above used notation, $\left((\mathcal{F})_{j}\right)_{\eta}$ stands for the value of the diagnosis that is in the $\mathrm{j}$-th vector of the statistical file $\mathcal{F}$ i.e. $\left((\mathcal{F})_{j}\right)_{\eta} \in \boldsymbol{\eta}$. )

Then, $P \eta \mid C_{k_{0}}(\mathbf{v})(d \mid \mathbf{v})$ is defined by

$$
P \eta \mid C_{k_{0}}(\mathbf{v})(d \mid \mathbf{v})=D\left(\mathcal{F}, \mathbf{v}, k_{0}, d\right) /\left(|\mathcal{F}| \cdot P\left(C_{k_{0}}(\mathbf{v})\right)\right)
$$

If $\mathbf{v}=\left(s_{1}, s_{2}, \cdots s_{n}\right) \in \boldsymbol{\Xi}$, we may approximate the conditional probability $P_{\eta \mid \xi_{1} \xi_{2} \ldots \xi_{n}}\left(d \mid s_{1}, s_{2} \cdots s_{n}\right)$ appearing in formula (1) by the $P \eta \mid C_{k_{0}}(\mathbf{v})(d \mid \mathbf{v})$ so that $P_{\eta \mid \xi_{1} \xi_{2} \ldots \xi_{n}}\left(d \mid s_{1}, s_{2} \cdots s_{n}\right)=P \eta \mid C_{k_{0}}(\mathbf{v})(d \mid \mathbf{v})$ and formula (1) can be applied as the decision rule in the SP algorithm.

The algorithm SP is presented in a symbolic programming language in section 4.2. The complexity of the algorithm SP will be defined, in section 5 , as a function of size $|\mathcal{F}|$ of the data file $\mathcal{F}$ and as a function of number $n$ of symptom variables. The complexity is verified on real data by measuring time required for making decision for one person.

The decision quality (or discernment power) is dealt with in section 6. In principle, it is the number of wrong classifications what is measured. However, it may be defined more formally:

Let $\mathcal{L} \subset \mathcal{F}, \mathbf{v} \in \boldsymbol{\Xi}$. Further, let $\operatorname{SP}(\mathcal{L}, \mathbf{v}) \in \boldsymbol{\eta}$ denote decision of SP when evidence (about a patient) is $\mathbf{v}$ and algorithm SP has the "learning" file $\mathcal{L}$ at his disposal. Then, "discernment power" of SP can be measured by percentage of wrong classifications either as

$$
100\left[1-1 /|\mathcal{F}| \sum_{j=1}^{|\mathcal{F}|} \delta\left(\operatorname{SP}\left(\mathcal{F},\left((\mathcal{F})_{j}\right)_{\Xi}\right),\left((\mathcal{F})_{j}\right)_{\eta}\right)\right]
$$

or with the formula

$$
100\left[1-1 /|\mathcal{F}| \sum_{j=1}^{|\mathcal{F}|} \delta\left(\operatorname{SP}\left(\mathcal{F} \backslash(\mathcal{F})_{j},\left((\mathcal{F})_{j}\right)_{\Xi}\right),\left((\mathcal{F})_{j}\right)_{\eta}\right)\right]
$$

This second approach is referred to as "Leave one out" technique. (There are more details on the technique in section 6.)

The results will be compared with one simple algorithm using the "marginal approach" in section 6.

\section{Detailed description of SP}

There is a theory of SP in section 3 and there is a realization of SP in this section 4. 
As mentioned above in section 2, SP algorithm is described twice. Namely, via a flowchart (see Figure 1.) and via a code written in a symbolic language.

Strictly speaking, realization of an algorithm is an executable file (i.e with extension .exe ) that is "understood" e.g. by any PC.

Less strictly, realization of an algorithm is syntactically and semantically correct text file "written" by a man-programmer in a general purpose programming language (like Fortran, $\mathrm{C}$ ,C++, Visual Basic) that is "understood" by a compiler of the respective language. (This text file has extensions like .for, .c, .vbs.)

Next level is symbolic language. It is an abstraction of general purpose languages. It is "written" by man and it can be "understood" by another man to the extent that he/she can rewrite it to correct text in general purpose language.

The last common representation of an algorithm is its flowchart. It is a "text" written by man-programmer in graphical language form. It has to be "understood" by another man. It has conventional structures like action blocks (rectangles), decision blocks (rhombus or trapezoid) and lines where arrows determine the "flow" of the program. Some symbols are overloaded. E.g. symbol "=" is interpreted as an assignment in action blocks whereas it is a relational operator in decision blocks.

To summarize, there is always potential place left for ambiguity and misunderstanding. Therefore, both descriptions of the realization are used in parallel and they are supplemented by informal comments that explain semantics of used structures (in context of theoretical foundations of the algorithm).

There is a slight functional difference between the flowchart and the symbolic language descriptions of the SP algorithm.

The flowchart describes entering one statistical file $\mathcal{L}$ (from the problem area) and entering a sequence of several new evidences $\mathbf{v}$ for which their optimal diagnoses are calculated.

In the symbolic language, there is a body of a function SP where only one given statistical file $\mathcal{L}$ and only one evidence $\mathbf{v}$ are entered as parameters.

Then, the corresponding diagnosis $d_{o p t}(\mathbf{v})$ is calculated and returned when the function SP ends. The difference is only a formal one and the essence of SP algorithm is the same.

\subsection{Flowchart of SP}

It represents the activity of the algorithm in a simplified form. It is a link between the theory and the realization. Therefore, the symbols from both "worlds" are used. E.g. the first action block describes the filling of the array $\mathrm{L}$ with values from the statistical file denoted as $\mathcal{L}$ to stress it is for "learning". Names of the symptoms, usually in form of text strings, are densely coded into integers. Denotation $\mathrm{L}[0-n, 1-|\mathcal{L}|]$ says that two-dimensional array $\mathrm{L}$ has $n+1$ positions in the first dimension. (Position " 0 " is used for storing diagnoses, positions " 1 " to "n" are for storing values of symptom variables). The second dimension is for indexing individual records from $\mathcal{L}$. Variable maxcount corresponds to $k_{0}$ via definition $k_{0}=n-$ maxcount. There are three loops in flowchart which are realized as for-cycles in the symbolic language. Filling the two-dimensional array LD with zeroes takes place anytime new evidence $\mathbf{v}$ is entered. In the end, after running through the whole $\mathrm{L}$ matrix, the array element $\mathrm{LD}(\mathrm{i}, \mathrm{j})$ contains the number $D\left(\mathcal{L}, \mathbf{v}, n-i, d_{j}\right)$. It is easy to see that elements of the matrix LD are integers and their sum is $|\mathcal{L}|$.

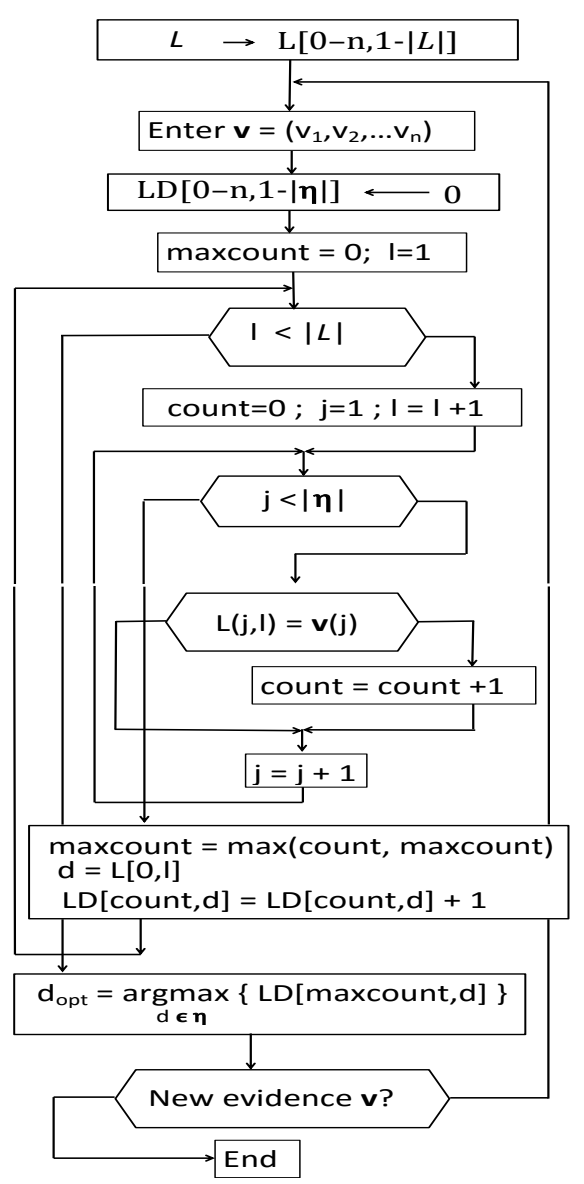

Figure 1. Flowchart of SP.

It would be possible to calculate the approximation of conditional probability $P \eta \mid C_{k_{0}}(\mathbf{v})(d \mid \mathbf{v})$ of $\eta$ on $C_{k_{0}}(\mathbf{v})$. (It can be done by normalization of the row $\mathrm{LD}$ (maxcount, ${ }^{*}$ ) of the array LD. i.e. by dividing elements in the row $\mathrm{LD}$ (maxcount,*) by their sum.) However, as we are interested only in the ranking, this normalization is not necessary and we look for $d_{o p t}$ by looking for maximal value in the row $\mathrm{LD}$ (maxcount, ${ }^{*}$ ) of matrix $\mathrm{LD}$, only. That way, we save computational time by skipping unnecessary actions.

Semantics of LD (level distance) can be seen from the fact that the row $\operatorname{LD}\left(\mathrm{i},{ }^{*}\right)$ contains number of all records/vectors from $\mathcal{L}$ that have just $i$ mutual symptom variables with the evidence $\mathbf{v}$. In other words, they belong to the equivalence class $C_{n-i}(\mathbf{v})$. 


\subsection{Description of SP in a symbolic language}

$S P$ algorithm can be used in different roles. It may be a simple "one-shot" decision-making, repeated decisionmaking for different apertures (sets of symptom variables whose values are disclosed to $S P$ as evidence), using $S P$ in a general testing scheme or it may be adapted to specific testing via "Leave one out" technique.

Instead of using one highly parametrized form of $S P$ algorithm, it seems better, for didactical reasons, to use several stand-alone modifications. However, only the most simple version, under the name function $S P$, will be presented in this paper. Specific modifications built on its basis ( and entitled SPL and SPA) will be mentioned in other sections. The following symbolic description is kept as simple as possible.

First, though the variables have their specific denotation reflecting their semantics, they are coded as integers or arrays of integers to make $S P$ faster.

Second, tests and resulting exceptions in inconsistent situations such as $|\mathcal{L}|=0$ or $|\boldsymbol{\eta}|=0$ are omitted !

Third, this version of $S P$ algorithm is defined for all $n$ available symptom variables. However, it can be easily modified if not values of all symptom variables are known, but if only some of them are provided as evidences (i.e. in case of smaller aperture).

Function SP returns the value $d_{o p t}(\mathbf{v})$ for each given $\mathbf{v}=$ $\left(v_{1}, v_{2} \cdots v_{n}\right) \in \boldsymbol{\xi}_{\mathbf{1}} \times \boldsymbol{\xi}_{\mathbf{2}} \times \ldots \boldsymbol{\xi}_{\boldsymbol{n}}$

1 function $\mathbf{S P}(\mathbf{v})$

$\mathbf{l . 1}$ expresses that $S P$ is a function $S P: \boldsymbol{\Xi} \longrightarrow \boldsymbol{\eta}$ i.e. accepts as argument the vector $\mathbf{v}$ and returns the optimal diagnosis $d_{\text {opt }}(\mathbf{v})$.

1.2 learning file $\mathcal{L}$ is stored in an array L. The value " 0 " in first dimension is for values of $\eta$.

1.3 - 1.7 sets zero values to the array LD (level distance) where metrics will be stored in the sequel.

1.9 - 1.21 For each $l \in \mathcal{L}$, number of symptom variables with coinciding values (symptoms) is calculated in the variable count. Increasing $\operatorname{LD}($ count, $\eta(1))$ by one increases chances of diagnosis $\eta(1)$ to become optimal $d_{\text {opt }}$ if the decision should take place at the level count.

l.16 - $\mathbf{l . 1 8}$ stores in maxcount the up-to-now achieved maximal number of coincidences.

1.23 - 1.30 finds in $\mathrm{LD}$ (maxcount,j) such diagnosis $d_{j}$ that would, on the level maxcount, define the winning dignosis $d_{\text {opt }}$. Naturally, if the number of cases from $\mathcal{L}$ is small (and that would result in objections from statistical point of view), it is possible to perform search for optimal diagnosis on a level count smaller than maxcount that would have more objects than level maxcount. Or even, it is possible to sum $L D(c t, j)$ for $c t=$ count to maxcount in an array $\mathrm{D}(1-|\boldsymbol{\eta}|)$ and search for $d_{o p t}$ in this array. (However, this modification of $S P$ is not available in the presented version.)

The link of the code with previous formal description may be made more clear if we realize that the variable maxcount is related to $k_{0}$. However, their roles are reversed in the sense that maxcount is the number of coincidences (of symptoms from $\mathbf{u}$ and $\mathbf{v}$ ) and should be as close to $n$ as possible, whereas $k_{0}$ is proximity (in the sense of the metrics $\rho$ ) and should tend to zero. Further, $\mathbf{v}(j)$ is $(\mathbf{v})_{j}$ and the value in the array $L D$ (maxcount,$j)$ stores the $D\left(\mathcal{L}, \mathbf{v}, k_{0}, d_{j}\right)$.

\section{Computational complexity}

It should be mentioned that experimenting with algorithms was performed on a statistical file $\mathcal{F}$, from the field of rheumatology with 1089 patients. Diagnosis variable $\eta$ contained 4 diagnosis and there were 34 symptom variables whose ranges had cardinalities from 2 to 9 . That way, no generation of artificial examples was necessary. Nevertheless, this choice has no influence on the substance of $S P$. Complexity $C_{S P}$ of $S P$ algorithm can be mesured with respect to the number of symptom variables $n$, number $|\mathcal{L}|$ of objects in the learning file $\mathcal{L}$ and with respect to the number $|\boldsymbol{\eta}|$ of diagnoses i.e. $C_{S P}=C(n,|\mathcal{L}|,|\boldsymbol{\eta}|)$. Due to the simple structure of $S P, C_{S P}$ can be estimated directly:

$$
\begin{array}{ll}
1.2 & c_{1} * n *|\mathcal{L}| \\
1.3-1.7 & c_{2} * n *|\boldsymbol{\eta}| \\
1.9-1.15 & c_{3} *|\mathcal{L}| *\left(n+c_{4}\right) \\
1.23-1.29 & c_{5} *|\boldsymbol{\eta}|
\end{array}
$$$$
C_{S P}=n\left[\left(c_{1}+c_{3}\right)|\mathcal{L}|+c_{2}|\boldsymbol{\eta}|\right]+c_{3} c_{4}|\mathcal{L}|+c_{5}|\boldsymbol{\eta}|
$$ 
Table 1. Computational time dependence on length $m=|\mathcal{F}|$ of file $\mathcal{F}$

\begin{tabular}{||c|r|r|r||}
\hline \hline $\mathrm{m}$ & $T_{\text {read }}$ & $T_{\text {total }}$ & $T_{\text {decision }}$ \\
\hline \hline 1000 & $31 \mathrm{msec}$ & $1 \mathrm{sec}$ & $10 \mathrm{msec}$ \\
\hline 2000 & $60 \mathrm{msec}$ & $2 \mathrm{sec}$ & $20 \mathrm{msec}$ \\
\hline 5000 & $156 \mathrm{msec}$ & $25 \mathrm{sec}$ & $50 \mathrm{msec}$ \\
\hline 10000 & $343 \mathrm{msec}$ & $100 \mathrm{sec}$ & $100 \mathrm{msec}$ \\
\hline 20000 & $687 \mathrm{msec}$ & $400 \mathrm{sec}$ & $200 \mathrm{msec}$ \\
\hline \hline
\end{tabular}

Table 2. Computational time dependence on width $n$ of file $\mathcal{F}$

\begin{tabular}{||c|r|r|r||}
\hline \hline $\mathrm{n}$ & $T_{\text {read }}$ & $T_{\text {total }}$ & $T_{\text {decision }}$ \\
\hline \hline 35 & $31 \mathrm{msec}$ & $0.546 \mathrm{sec}$ & $0.5 \mathrm{msec}$ \\
\hline 70 & $31 \mathrm{msec}$ & $1.046 \mathrm{sec}$ & $0.9 \mathrm{msec}$ \\
\hline 105 & $47 \mathrm{msec}$ & $1.516 \mathrm{sec}$ & $1.3 \mathrm{msec}$ \\
\hline 140 & $45 \mathrm{msec}$ & $1.968 \mathrm{sec}$ & $1.8 \mathrm{msec}$ \\
\hline 200 & $78 \mathrm{msec}$ & $2.78 \mathrm{sec}$ & $2.59 \mathrm{msec}$ \\
\hline 300 & $109 \mathrm{msec}$ & $4.07 \mathrm{sec}$ & $3.78 \mathrm{msec}$ \\
\hline \hline
\end{tabular}

The assumption of linearity $\left(c_{1}, c_{2} \cdots\right)$ is a bit simplifiction and valid only for small ranges of $n,|\mathcal{L}|,|\boldsymbol{\eta}|$. If the ranges are greater, then effects like "paging" of memory, the way files are stored in a concrete file system (e.g. FAT 32 or NTFS ) and variables used for storing the "coding" numbers may come in play. E.g. values of $\boldsymbol{\eta}$ are stored in variables of type integer*1 and therefore should not exceed 256.

Therefore, instead of looking for explicit values for $c_{1}, \cdots c_{5}$, direct measurements are documented in Table 1 where length $|\mathcal{L}|$ of $\mathcal{L}$ varies from 1000 to 20000 and in Table 2 where width $n$ of $\mathcal{L}$ varies from 35 to 300 . Corresponding files $\mathcal{L}$ (e.g. $\mathcal{L}(70,1089)$ or $\mathcal{L}(35,20000))$ were generated from original $\mathcal{L}$ (i.e. $\mathcal{L}(35,1089)$ ) by repeating respective rows and columns. In the Tables 1 and 2 , column $T_{\text {read }}$ contains time necessary to read $\mathcal{L}$. Column $T_{\text {total }}$ increases with square power of $|\mathcal{L}|$ as it is the time necessary for $|\mathcal{L}|$ decisions. When $T_{\text {total }}$ is divided by $|\mathcal{L}|$, then the times in column $T_{\text {decision }}$ are always below $1 \mathrm{sec}$ and therefore completely acceptable. Based both on analysis and direct measurements, complexity of $S P$ is not a problem. Therefore, the limiting factor for better discernment power of $S P$ is an externality i.e. experts should provide bigger data in form of a file $\mathcal{F}$ (or $\mathcal{L})$.

\section{Simulation results}

This section should reflect the decision-making quality of $\mathrm{SP}$ via simulation results. Simulation is performed on the data mentioned in the beginning of section 5. Though the following example is very simple, it may reveal interesting facts when comparing $S P$ and the decision-making algorithm $A 4$ (from [7] and mentioned in [8]) that can serve as a simple representative of marginal-based algorithms. Let knowledge base $\mathcal{K B}$ consist of 3 marginals i.e. $\mathcal{K B}=\left\{m_{1}, m_{2}, m_{3}\right\}$.

The marginals can be defined by their "generating" symptom variables (besides implicitely supposed diagnosis variable $\eta$ ). Underscoring of symbol for a marginal denotes the set of "generating" symptom variables. E.g., if $\underline{m_{1}}=$ $\left\{\xi_{25}, \xi_{33}\right\}$, then $m_{1}=P_{\eta \xi_{25} \xi_{33}}$.

Then, $\mathcal{K B}$ used for testing was defined via

$$
\underline{m_{1}}=\left\{\xi_{25}, \xi_{33}\right\}, \underline{m_{2}}=\left\{\xi_{26}, \xi_{32}\right\}, \underline{m_{3}}=\left\{\xi_{27}, \xi_{33}\right\}
$$

The "testing environment" provides an easy way to manipulate with "inputs" to the decision-making algorithm.

First, it makes possible to remove marginals from $\mathcal{K B}$ and second, not all symptom variables, from $n$ possible ones, need to be revealed as "evidences" to decision makingalgorithm $S P$ or $A 4$. We tested $S P$ or $A 4$ alongside for 8 different situations $s_{1}, s_{2}, \cdots s_{8}$ described in Table 3. E.g., the expression $\left\{m_{1}, m_{3}\right\} \cap\left\{\xi_{1}-\xi_{33}\right\}$ (in column "marginals $\cap$ variables" of Table 3 ) stands for situation $s_{2}$ where $\mathcal{K B}$ consists only of marginals $\left\{m_{1}, m_{3}\right\}$ and values of all 33 symptom variables $\left\{\xi_{1}-\xi_{33}\right\}$ are submitted as "evidences" to the $S P$ and $A 4$. (Naturally, $\left\{m_{1}, m_{3}\right\}$ has impact only on $A 4$, whereas $\left\{\xi_{1}-\xi_{33}\right\}$ influences both $S P$ and $A 4$ ). Column "active variables" in Table 3 contains symptom variables whose values have influence on $A 4$ as result of both conditions. Column "active space" is product of their ranges. As all symptom variables here are dichotomical ones, the values are like $4,16,32$.

Even the above mentioned denotation for individual marginals is a little simplified. E.g. $m_{1}=P_{\eta \xi_{25} \xi_{32}}$ is not enough as it should be also mentioned what data was used for populating the marginal $m_{1}$. This can be expressed by adding the source. E.g. $m_{1}=P_{\eta \xi_{25} \xi_{32}}(\mathcal{L})$ stands for the marginal filled from the data set $\mathcal{L}$. This denotation would do for the column $A 4 A$, but not for calculating the values for column $A 4 L$. Then, in fact, there are 1089 different marginals $m_{1}(\mathcal{L} \backslash t)=P_{\eta \xi_{25} \xi_{32}}(\mathcal{L} \backslash t)$. Those marginals will be populated from 1089 different data files $\mathcal{L} \backslash t$ ) that have to be created just for the purpose.

The "A" (in column $A 4 A$ containing number of wrong classifications) stresses that all data was used both for learning and testing i.e. $\mathcal{L}=\mathcal{T}=\mathcal{F}$. The "L" (in column $A 4 L$ ) has the meaning that the method "Leave one out" was used for the calculation of "discernment power" of algorithm A4. 
The essence of "Leave one out" technique consists in following steps:

1. The $j$-th vector from file $\mathcal{F}$ (denoted as $\left.(\mathcal{F})_{j}\right)$ is removed from $\mathcal{F}$.

2. The resulting learning file $\mathcal{L}_{j}=\mathcal{F} \backslash\left\{(\mathcal{F})_{\mid}\right\}$is used for populating all necessary marginals in $\mathcal{K B}$.

3. The "symptom part" $\left((\mathcal{F})_{j}\right)_{\boldsymbol{\Xi}}$ of vector $(\mathcal{F})_{j}$ is submitted as input to the decision-making algorithm (e.g. $S P$ or $A 4$ ).

4. The resulting decision (e.g. $\left.A 4\left(\left((\mathcal{F})_{j}\right)_{\Xi}\right)\right)$ is compared with the "diagnosis part" $\left((\mathcal{F})_{j}\right)_{\eta}$ of vector $(\mathcal{F})_{j}$ and coincidence of those two diagnoses is "marked" as 1 .

5. The same procedure is repeated $|\mathcal{F}|$ times (for $j=$ $1,2, \ldots|\mathcal{F}|)$ and the normalized sum of coincidences is an indicator of decision quality of the algorithm. Instead of coincidences, we prefer to use percentage of misclassifications as can be seen in second formula in Section 2

It can be observed in Table 4 that L-values are higher than corresponding A-values. In general, $S P$ is slightly better than $A 4$, but not always

e.g. $A 4 L(s 8)=423<S P L(s 8)=431$. On the basis of other similar experiments, it looks like that advantages of $S P$ may be more prominent but only for A-testing. Especially for $\mathcal{K B}$ with more marginals and when values of all symptom variables are known. As far as "Leave one out" method is concerned and not with full-sized evidence, no decisive conclusions can be drawn, so far. However, it seems that $S P$ does quite well and could be used along with other recommended methods.

\section{Discussion and conclusions}

Among positive features of marginal-less $S P$ algorithm, the following ones can be mentioned :

1. The presented algorithm $S P$ is sufficiently fast i.e. decisions are made within seconds.

2. $S P$ has good discernment power when the tested case $t$ was included in the learning file $\mathcal{L}$ and values of all symptom variables (from $\mathcal{L}$ ) are given as input evidence.

3. It is easy to add new cases (or remove old ones if considered as obsolete) to the learning file $\mathcal{L}$. In marginal-based approach, it is necessary to recalculate the marginals.
4. Problems associated with selection of marginals are avoided (by definition !) and only symptom variables are necessary. In general, values of all symptom variables (present in the learning file $\mathcal{L}$ ) should be provided as evidences, if available.

5. Testing via "Leave one out" technique is extremely easy with a small modification in the presented code of $S P$. It takes approximately the same time as testing on the all data (i.e. when $\mathcal{L}=\mathcal{T}$ ). Marginal-based algorithm require for "Leave one out" a lot of time for splitting the data file $(|\mathcal{F}|)$ times !) and filling the marginals for each split.

6. If an evidence $\mathbf{v} \in \boldsymbol{\Xi}$ turns up, as input for $S P$, that is not present in the learning file $\mathcal{L}$ i.e. $\mathbf{v} \notin\left((\mathcal{F})_{j}\right) \mathbf{\Xi} \quad$ for $j=1,2, \cdots|\mathcal{F}|$ then $S P$ recommends the diagnosis with the highest apriori probability i.e.

$$
S P(\mathbf{v})=\underset{d_{j} \in \boldsymbol{\eta}}{\operatorname{argmax}} P_{\eta}\left(d_{j}\right)
$$

what sounds as one would expect.

7. If there is a valid piece of knowledge of logical nature (e.g. implications), it is possible to force it out just by repeating artificial records representing it in $\mathcal{L}$ several times.

$S P$ has several drawbacks as well:

1. $S P$ can be applied only to nominal variables (i.e. not continuous, not cardinal and even not to ordinal) due to properties of the proximity metrics $\rho$.

2. As the only testing criterion is number of wrong classifications, $S P$, in its presented version, is not a proper choice for risk analysis.

3. With decreasing number of symptoms (evidences), discernment power of $S P$ drops as well. (It is similar to marginal-based algorithms, as well.)

4. It is not possible to add additional knowledge about structure of $P$ (e.g. in form of graph models expressing conditional independence among marginals.) All is based on input data represented by the statistical file $\mathcal{F}$ (or $\mathcal{L}$ ) only.

There are some open questions to study.

One would expect that decision quality is decreasing with increasing value $k$ of proximity metrics $\rho$. It can be observed on testing runs. However, one would expect certain invariance or at least monotonicity in recommended $d_{o p t}$. In general, it does not always happen and recommended $d_{o p t}$ "oscillates" when moving in array LD to levels with smaller symptom incidence $i$ (or otherwise for $\rho$ higher $k$ value.). Would it be possible to change this behaviour via introducing a sort of "weighting" on symptom variables? Up to now, 
Table 3. Different testing situations

\begin{tabular}{||c|r|r|r||}
\hline \hline situations & marginals $\cap$ variables & active variables & active space \\
\hline$s_{1}$ & $\left\{m_{1}, m_{2}, m_{3}\right\} \cap\left\{\xi_{1}-\xi_{33}\right\}$ & $\xi_{25}, \xi_{26}, \xi_{27}, \xi_{32}, \xi_{33}$ & 32 \\
\hline$s_{2}$ & $\left\{m_{1}, m_{3}\right\} \cap\left\{\xi_{1}-\xi_{33}\right\}$ & $\xi_{25}, \xi_{27}, \xi_{33}$ & 4 \\
\hline$s_{3}$ & $\left\{m_{2}\right\} \cap\left\{\xi_{1}-\xi_{33}\right\}$ & $\xi_{26}, \xi_{33}$ & 4 \\
\hline$s_{4}$ & $\left\{m_{1}\right\} \cap\left\{\xi_{1}-\xi_{33}\right\}$ & $\xi_{25}, \xi_{33}$ & 4 \\
\hline$s_{5}$ & $\left\{m_{3}\right\} \cap\left\{\xi_{1}-\xi_{33}\right\}$ & $\xi_{27}, \xi_{33}$ & 16 \\
\hline$s_{6}$ & $\left\{m_{2}, m_{3}\right\} \cap\left\{\xi_{1}-\xi_{33}\right\}$ & $\xi_{26}, \xi_{27}, \xi_{32}, \xi_{33}$ & 16 \\
\hline$s_{7}$ & $\left\{m_{1}, m_{2}, m_{3}\right\} \cap\left\{\xi_{1}-\xi_{32}\right\}$ & $\xi_{25}, \xi_{26}, \xi_{27}, \xi_{32}$ & 2 \\
\hline$s_{8}$ & $\left\{m_{1}, m_{2}, m_{3}\right\} \cap\left\{\xi_{33}\right\}$ & $\xi_{33}$ & 4 \\
\hline
\end{tabular}

Table 4. Comparing wrong classifications for SP and A4

\begin{tabular}{||c|c|c|c|c||}
\hline \hline situations & SPL & SPA & A4L & A4A \\
\hline \hline$s_{1}$ & 421 & 415 & 427 & 421 \\
\hline$s_{2}$ & 462 & 460 & 463 & 462 \\
\hline$s_{3}$ & 538 & 538 & 538 & 538 \\
\hline$s_{4}$ & 464 & 464 & 464 & 464 \\
\hline$s_{5}$ & 463 & 461 & 463 & 461 \\
\hline$s_{6}$ & 431 & 420 & 423 & 422 \\
\hline$s_{7}$ & 537 & 530 & 539 & 539 \\
\hline$s_{8}$ & 464 & 464 & 464 & 464 \\
\hline \hline
\end{tabular}

all symptom variables were supposed as equally informative. And if it would be effective, how to implement it in the existing realization of the SP? Another topic that would deserve a deeper investigation into is behaviour of SP for small sets of disclosed symptom variables (so called aperture) used for defining evidence. Though the improvement in decision quality (with respect to alternative approaches) may be only several percentage points, it would be interesting to find if, on average, SP is a reliable tool for decision-making even in this situation.

To summarize, with respect to above mentioned arguments, $S P$ can be recommended for decision-making on nominal symptom variables and when a sufficient learning data file is available. It may serve as an alternative to well established marginal-based algorithms for decision-making under uncertainty.It has sound basic philosophy ("Patients with similar symptoms should have a similar diagnosis") and it is theoretically well founded (conditioning on equivalence classes induced by proximity metrics $\rho$ and direct link to the optimal solution represented by formula 1). According to simulations (described in section 6) on a realistic study case (mentioned in section 5), it does quite well and it does not require sophisticated suppositions (like maximal entropy principle or Markovian blanket) about structure of approximating joint distributions. The problem with marginal selection (i.e "how many" and "what composition" ) is circumvented in SP. Remark: The original version (entitled "Diagnostic problem without marginals") was published at Wupes 15 Workshop at Moninec. This is the extended version of that.

\section{Acknowledgements}

I am very grateful to the anonymous referee for valuable comments and suggestions to improve legibility of the paper. My thanks belong also to R.Jiroušek who drew my attention to the fact that suggested metrics can be seen as an instance of Hamming distance known from coding theory.

\section{REFERENCES}

[1] P.Cheeseman: A method of computing generalized Bayesian probability values of expert systems with probabilistic background, in: Proc. 6-th Joint Conf. on AI(IJCAI-83), Karlsruhe

[2] W.E. Deming, F.F Stephan: On a least square adjustment of sampled frequency table when expected marginal totals are known, Ann.Math.Stat. 11(1940), pp. 427 - 444

[3] E.T. Jaynes: On the rationale of maximum-entropy methods, Proc. of the IEEE 70 (1980), pp. 939 - 952.

[4] Terrence L.Fine: Theories of Probability: An examination of foundations, Academic Press,1973)

[5] David Kahnemann: Thinking: Fast and Slow, (2012)

[6] H.G. Kellerer: Verteilungsfunktionen mit gegebenen Marginalverteilungen. Zeitschrift für Wahrscheinlichkeitstheorie, 3(1964), pp. $247-270$. 
[7] O. Kř́ž: A new algorithm for decision making with probabilistic background, in: Transactions of the Eleventh Prague Conference on Information Theory, Statistical Decision Functions and Random Processes, August 27-31, 1990, Vol. B, (Academia,Prague, 1992) pp 135-143

[8] O. Kř́ž: Comparing algorithms based on marginal problem. Kybernetika, Vol 43, (2007), No.5, 633-647

[9] O. Kř́ž: Selecting marginals for decision making based on marginal problem. WUPES'09, Vol 43, (2009), No.5, 633647
[10] O. Kř́íz: Mixing marginals for decision making based on marginal problem. In: WUPES 2012, Proceedings of the 9th Workshop on Uncertainty Processing, (T. Kroupa,J. Vejnarová ed.), Mariánské Lázně 2012, pp. 114-125.

[11] A.Perez: A probabilistic approach to the integration of partial knowledge for medical decision-making (in Czech). In: Proc. of the 1-st Czechoslovak Congress of Biomedical engineering (BMI83) (J. Zvárová ed.), Mariánské Lázně 1983, pp. 221-226. 